\title{
Biodegradable polymeric prodrugs of naltrexone
}

\author{
D.B. Bennett ${ }^{1}$, X. Li $^{1}$, N.W. Adams ${ }^{1}$, S.W. Kim ${ }^{1}$, C.J.T. Hoes ${ }^{2}$ and J. Feijen ${ }^{2}$ \\ ${ }^{1}$ Department of Pharmaceutics, University of Utah, Salt Lake City, UT (U.S.A.) and ${ }^{2}$ Department of Chemical Technology, \\ University of Twente, Enschede (The Netherlands)
}

(Accepted 15 August 1990)

The development of a biodegradable polymeric drug delivery system for the narcotic antagonist naltrexone may improve patient compliance in the treatment of opiate addiction. Random copolymers consisting of the $\alpha$-amino acids $N^{5}$-(3-hydroxypropyl)-L-glutamine and L-leucine were synthesized with equimolar initial monomer feeds. The molecular weight of this chemical carrier was determined by viscometry and wide-angle light scattering. In order to get selective covalent coupling of drug to polymer the 3-acetate derivative and the 14-acetate derivative of naltrexone were synthesized and characterized by NMR. Hydrolytic conversion of each monoacetate to parent drug was monitored by HPLC and the rate constant was determined. Both derivatives were coupled via hydrolytically labile carbonate linkages to the polymer hydroxyl groups. The drug conjugates were prepared as particles of various size ranges between 20 and $350 \mu$. In vitro studies in phosphate-buffered saline (pH 7.4) demonstrated a release rate dependence on particle size. Nearly constant plasma levels of naltrexone were obtained for one month after subcutaneous injection in rats.

Keywords: Biodegradable; Naltrexone; Narcotic antagonist; Poly ( $\alpha$-amino acids)

\section{Introduction}

Opiate addiction presents a significant problem to the health, safety and economic condition of many nations. For the treatment of opiate addicts naltrexone hydrochloride is used due to its ability to antagonize opiates. Poor compliance is a major difficulty in patients receiving naltrexone. Naltrexone does not directly relieve unpleasant symptoms such as prolonged withdrawal or drug seeking behavior. Therefore, compliance becomes more tenuous $[1,2]$.

The development of a long-term controlled release system for the delivery of naltrexone has

Correspondence to: J. Feijen, Department of Chemical Technology, University of Twente, P.O. Box 217, 7500 AE Enschede, The Netherlands. been sought to overcome the problems associated with compliance over several months or years of therapy. The National Institute on Drug Abuse has determined that a biodegradable polymeric delivery system that releases naltrexone at a constant rate is most desirable [3] .

The aim of our research is to develop an injectable system of polymeric prodrug particles which will release constant and effective levels of naltrexone in vivo for at least one month. In previous studies we described the preparation and drug release characteristics of insoluble macromolecular prodrugs of naltrexone bound to biodegradable polymers $[4,5]$. As carriers the polymer of $N^{5}$-(3-hydroxypropyl)-L-glutamine (HPG) and copolymers of HPG and leucine (Leu ) were used. Naltrexone-3-acetate-14- 
hemisuccinate was bound across an ester bond with the hydroxyl groups of the polymeric carrier. Conjugates were prepared both as discs and as particles. By increasing the content of hydrophobic Leu residues in the polymeric carrier up to $50 \%$ the duration of in vitro naltrexone release from particle-shaped conjugates could be increased to 15 days and acceptable plasma levels were obtained after subcutaneous injection in rats [5]. To enhance the duration of naltrexone release from particle-shaped polymeric conjugates we now have studied the use of a different type of drug-polymer bond [6]. In this paper we report on particle-shaped naltrexone conjugates of $\mathrm{P}\left(\mathrm{HPG}^{50} / \mathrm{Leu}^{50}\right)$ in which the drug is bound at either the 3-position or the 14-position through a carbonate bond. The preparation and in vitro as well as in vivo drug release characteristics are described.

\section{Experimental}

\section{Materials}

All materials were of reagent grade quality or better and used as received except as noted. Dioxane, tetrahydrofuran (THF), and absolute ethanol were stored over $4-\AA$ molecular sieves before use. Triethylamine (TEA), dimethylformamide (DMF), and acetic anhydride were distilled prior to use. Triophosgene was obtained from Janssen (Belgium ). Naltrexone hydrochloride was obtained from the Research Triangle Institute and converted to the free base form (Nf) before further modification [4].

\section{Methods}

Thin-layer chromatography (TLC) was performed using silica gel 60F-254 precoated plastic or aluminum sheets (Merck) with chloroform/ methanol (4:1) as developer and visualized with UV light and Dragendorff's reagent (Sigma Chemical Co.). High performance liquid chromatography (HPLC) was performed by using a mobile phase of $60 \%$ methanol and $40 \%$ aqueous $(0.25 \%$ TEA, $0.01 \%$ sodium octylsulfate, $\mathrm{pH}$ adjusted to 6.7 with phosphoric acid) with a $\mu$ Bon- dapak C-18 $(30 \mathrm{~cm}$ ) column (Waters Associates), and a Spheri-5 C-18 guard column (Brownlee Labs) at a flow rate of $1.0 \mathrm{ml} / \mathrm{min}$. Samples (usually $30 \mu \mathrm{l}$ ) were injected, the UV absorbance at $254 \mathrm{~nm}$ detected, and peak heights recorded. Quantitation was done by comparison of peak heights to a standard curve.

Viscometric measurements were made at $25.0^{\circ} \pm 0.1^{\circ} \mathrm{C}$ in dichloroacetic acid (DCA) with an Ubbelohde viscometer. Initial polymer concentration was $0.2 \%(\mathrm{w} / \mathrm{v})$ with two subsequent dilutions. Efflux time for solvent was $412 \pm 1 \mathrm{~s}$. Comparison of intrinsic viscosities with the literature for HPG containing polymers yields the viscosity based molecular weight (Mv).

Polymer samples for analysis by wide-angle light scattering (WALS) were prepared at various concentrations in dry DCA, usually $0.4,0.3$, 0.2 , and $0.1 \%(\mathrm{w} / \mathrm{v})$. Solutions were filtered through a $0.2 \mu \mathrm{m}$ membrane to ensure complete removal of dust and fine particulates. The intensity of scattered light at angles ranging from $30^{\circ}$ to $150^{\circ}$ was determined using a FICA 50 WALS instrument with a mercury lamp ( $546 \mathrm{~nm})$. Refractive indices of the copolymer/DCA solutions were measured at $25^{\circ} \mathrm{C}$ with a Brice-Phoenix differential refractometer with a mercury lamp (546 nm ). Construction of a Zimm plot allows for extrapolation of the inverse of the weight-averaged molecular weight ( $\mathrm{Mw})$.

${ }^{1} \mathrm{H}$ and ${ }^{13} \mathrm{C}$ NMR spectra were obtained using a JEOL HNM-FX $270 \mathrm{MHz}$ Fourier transform NMR spectrometer. Naltrexone derivatives were dissolved in $\mathrm{CDCl}_{3}$ and chemical shifts $(\delta, \mathrm{ppm})$ were referenced to internal tetramethylsilane (TMS). IR spectra were recorded using a Beckman Microlab $620 \mathrm{MX}$ computing spectrometer. Naltrexone and its derivatives were dissolved in acetone and allowed to dry on sodium chloride discs. Melting points were determined using a hot block Mel-Temp capillary melting point apparatus and are uncorrected.

\section{$\operatorname{Copoly}(\alpha$-amino acid $)$ carriers}

Random copolymers of HPG and Leu are obtained by a well known method involving the displacement of the $\gamma$-benzyl group from copol- 
ymers of $\gamma$-benzyl-L-glutamate (BLG) and Leu by aminolysis [4-9]. The copoly (BLG/Leu) was synthesized utilizing equimolar quantities of the amino acid $N$-carboxyanhydride monomers in dioxane with TEA as catalyst $(A / I=35)$ according to the general method of Blout et al. [10] as described in our previous study [4]. Treatment of this copolymer with 3-aminopropanol for 5 days produces the HPG/Leu copolymer with fewer than 3\% residual benzyl groups remaining $[7,8]$. Molecular weight was determined by both viscometry and wide-angle light scattering (WALS) analyses.

\section{Naltrexone monoacetate prodrugs}

Naltrexone-3-acetate (N3A) was synthesized according to a modification of a previously reported procedure [4]. Nf (3.07 g) was reacted at $0-5^{\circ} \mathrm{C}$ with acetic anhydride $(1.1 \mathrm{ml}, 1.3 \mathrm{~mol}$ eq. $)$ in THF $(20 \mathrm{ml})$ with TEA (3.0 mol eq.) present as catalyst. After stirring overnight the solution was concentrated under high vacuum. The semi-solid product was purified by flash chromatography using $2 \%$ methanol in chloroform as eluent and silica gel 60 as stationary phase. Subsequent crystallization from methylene chloride/hexane gave N3A (3.21 g, 93.3\%) after filtering and drying under vacuum; m.p. $103-105^{\circ} \mathrm{C}$; TLC, $R_{\mathrm{f}} 0.84$; HPLC, $R_{\mathrm{t}} 11.9 \mathrm{~min}$; ${ }^{\prime} \mathrm{H}$ NMR $\delta, 6.86,6.82,6.69,6.65$ (H-1, H-2 AB quartet), 4.69 (H-5), 3.21 (H-9), and 2.31 (3acetate methyl $\mathrm{H}$ ); ${ }^{13} \mathrm{C}$ NMR $\delta, 20.80$ (3-acetate methyl C), 168.42 (3-acetate carbonyl C); IR, $1730 \mathrm{~cm}^{-1}$ (6-keto), $1770 \mathrm{~cm}^{-1}$ (3-acetate).

Naltrexone-14-acetate (N14A) was synthesized by the selective deacylation of naltrexone3,14-diacetate (N3,14A) [11]. Nf (1.28 g) was refluxed in $25 \mathrm{ml}$ freshly distilled acetic anhydride at $135^{\circ} \mathrm{C}$ for $5 \mathrm{~min}$ and the temperature was decreased to $130^{\circ} \mathrm{C}$. After $25 \mathrm{~min}$ the solution was allowed to cool gradually to room temperature and was concentrated by rotary evaporation. The $\mathrm{pH}$ of the residue was adjusted to a value of about 8 with prechilled $5 \%$ sodium bicarbonate. Extraction into methylene chloride $(50 \mathrm{ml})$ twice, evaporation, and crystallization from toluene/hexane yielded $1.26 \mathrm{~g}$ of $\mathrm{N} 3,14 \mathrm{~A}$
(78.7\%); m.p. $144.5-146^{\circ} \mathrm{C}$ (see lit. in [11] $146-147^{\circ} \mathrm{C}$ ); TLC, $R_{\mathrm{f}} 0.93 ;$ HPLC, $R_{\mathrm{t}} 14.7 \mathrm{~min}$; ${ }^{1} \mathrm{H}$ NMR $\delta, 6.87,6.83,6.70,6.66(\mathrm{H}-1, \mathrm{H}-2 \mathrm{AB}$ quartet), 4.69 (H-5), 4.47 (H-9), and 2.31 (3acetate methyl $\mathrm{H}$ ), 2.19 (14-acetate methyl $\mathrm{H}$ ); ${ }^{13} \mathrm{C}$ NMR $\delta, 20.73$ (3-acetate methyl C), 168.27 (3-acetate carbonyl C), 22.14 (14-acetate methyl C), 170.03 (14-acetate carbonyl C); IR, 1735 $\mathrm{cm}^{-1}$ (6-keto, 14-acetate), $1765 \mathrm{~cm}^{-1}$ (3acetate ).

Selective deacetylation of $\mathrm{N} 3,14 \mathrm{~A}(1.25 \mathrm{~g})$ in $4 \%$ sulfuric acid $(40 \mathrm{ml})$ overnight at room temperature was followed by basification and extraction with methylene chloride. The organic layer was dried over anhydrous sodium sulfate for $12 \mathrm{~h}$ at $4^{\circ} \mathrm{C}$, filtered, and methylene chloride was evaporated under vacuum, giving a white residue. The crude product was crystallized from methylene chloride/hexane to give $0.75 \mathrm{~g}$ of N14A (66.0\%); m.p. $192-194^{\circ} \mathrm{C}$; TLC, $R_{\mathrm{f}} 0.87$; HPLC, $R_{\mathrm{t}} 10.8 \mathrm{~min} ;{ }^{1} \mathrm{H}$ NMR $\delta, 6.75,6.71,6.62$, 6.58 (H-1, H-2 AB quartet), 4.68 (H-5), 4.48 (H-9), and 2.21 (14-acetate methyl $\mathrm{H}$ ); ${ }^{13} \mathrm{C}$ NMR $\delta, 22.86$ (14-acetate methyl C), 170.11 (14-acetate carbonyl C); IR, $1725 \mathrm{~cm}^{-1}$ (6-keto, 14-acetate).

\section{In vitro hydrolysis of naltrexone prodrugs}

To demonstrate the facile conversion of the prodrug intermediates to naltrexone, in vitro hydrolysis studies were performed. The hydrolytic medium was phosphate-buffered saline (PBS, $\mathrm{pH}$ $\left.7.4,37^{\circ} \mathrm{C}\right)$. Typically, a prodrug sample $(10 \mathrm{mg})$ was dissolved in $200 \mu \mathrm{l}$ of ethanol and then added to PBS $(25.0 \mathrm{ml})$. Aliquots $(50 \mu \mathrm{l})$ were taken for various time intervals and quenched by rapid cooling with liquid nitrogen. Quantitation of naltrexone and the esters was accomplished by HPLC.

\section{Copoly( $\alpha$-amino acid)-naltrexone conjugates}

A solution of $0.50 \mathrm{~g} \mathrm{~N} 14 \mathrm{~A}$ in $10 \mathrm{ml}$ of chloroform was added drop-wise to $1.0 \mathrm{~g}$ of triphosgene in chloroform $(20 \mathrm{ml})$ and TEA $(0.7 \mathrm{ml})$ with stirring and was allowed to react for $1 \mathrm{~h}$ before an additional $1.4 \mathrm{ml}$ of TEA were added. After 4 
$h(\mathrm{~N} 14 \mathrm{~A})$ or $22 \mathrm{~h}(\mathrm{~N} 3 \mathrm{~A})$ reaction time the drug solution was evaporated to near dryness under a stream of dry nitrogen and a solution of $0.50 \mathrm{~g}$ of $\mathrm{P}\left(\mathrm{HPG}^{50} / \mathrm{Leu}^{50}\right)$ in $27 \mathrm{ml}$ of chloroform/DMF (5:4) was added at once, and the mixture was stirred for $40 \mathrm{~h}$. Solid polymer-drug conjugate was isolated by drop-wise addition of the reaction solution to $1800 \mathrm{ml}$ of ether, washed with $200 \mathrm{ml}$ ether, redissolved in $50 \mathrm{ml}$ of absolute ethanol, and reprecipitated in $1400 \mathrm{ml}$ of ether. The product was filtered and dried in vacuo to constant weight $(0.62 \mathrm{~g})$.

Both batches were ground into particles with a model CBM-1 (Black and Decker, Shelton, U.S.A.) electronic mill grinder. The powders were passed through sieves of predetermined mesh sizes to get various particle size ranges (20$50,50-100,100-200$, and $200-350 \mu \mathrm{m}$ ) to investigate particle size effects on drug release.

To determine the extent of drug loading polymer-drug conjugate samples of known weight were dissolved in $2 \%$ methanolic $\mathrm{KOH}$ and stirred overnight at room temperature. The amount of drug in solution was quantified by HPLC analysis and comparison to a standard curve. No degradation of naltrexone was seen in a control consisting of drug dissolved in the same solvent.

\section{In vitro release studies}

Polymeric prodrug samples of known weight and particle size were placed in dialysis bags (molecular weight cut-off $=3500$ ). The bags were filled with $250 \mu \mathrm{l}$ of phosphate-buffer saline (PBS, pH 7.4, 0.1\% $\mathrm{NaN}_{3}$ ) and tied such that a minimum of air remained inside. These bags were then immersed in $5.0 \mathrm{ml}$ of PBS within a silanized test tube with screw-top lids. The tubes were then placed in a shaker water bath $\left(37.0 \pm 0.3^{\circ} \mathrm{C}, 50\right.$ strokes $/ \mathrm{min}, 2.0 \mathrm{~cm} /$ stroke ). Samples were collected daily and the entire release medium was replaced with fresh PBS. Samples were stored at $4^{\circ} \mathrm{C}$ until naltrexone was quantified by HPLC analysis. Systems were studied in triplicate.

\section{In vivo release studies}

Female Sprague-Dawley rats (180-200 g) were chosen as the animal model because of their ease of handling and the precedent set in previous narcotic antagonist studies regarding the use of rats $[12,13]$. Rats were housed for the duration of the studies and were allowed to consume food and water ad libitum with 12-h periods of light and dark daily. At the conclusion of the experiments the rats were killed by carbon dioxide asphyxiation. Naltrexone concentration in rat plasma was determined by gas chromatography coupled with negative ion chemical ionization mass spectrometry [14].

Preweighed amounts of polymeric prodrug conjugate (between 10 and $32 \mathrm{mg}$ ) were placed in $1.0-\mathrm{ml}$ syringes with 18 -gauge needles. Rats were lightly anesthetized with ether and the hair around the site of injection shaved. Injection of the particles $(50-100 \mu \mathrm{m})$ as a suspension in sterile saline ( $<0.3 \mathrm{ml}$ total volume) was made into the dorsal side of the rat's neck. Any conjugate remaining in the needles and syringes was recovered, dried, and weighed. All studies were performed in triplicate, although periodically individual rats resisted sampling and thus were skipped.

The conjugates had all been washed with ethanol during their final preparations and were assumed to be sterile. However, to minimize any chance of infection tetracycline was included in the drinking water for the first week of the study. Blood was drawn by carefully snipping the very end $(<1 \mathrm{~mm})$ of the rat's tail which was then 'milked' for collection. Without the milking process, the blood flow stopped almost immediately. Samples (usually 50-500 $\mu$ l) were collected in Becton Dickinson Microtainer brand capillary whole blood collectors with EDTA as anticoagulant. Plasma was separated by centrifugation, stored in silanized tubes, and frozen at $-20^{\circ} \mathrm{C}$ until analyzed by GC/MS. Plasma concentrations are expressed as $\mathrm{ng} / \mathrm{ml} / \mathrm{mg}$ conjugate. 


\section{Results and discussion}

\section{Design characteristics of naltrexone delivery systems}

The ideal naltrexone delivery system would have the following characteristics: (i) administration by injection, (ii) no adverse tissue reaction, (iii) relatively constant drug release, and (iv) biodegradability. The biodegradable polymeric naltrexone delivery systems studied to date include many approaches using different copolymers, geometries, and drug loading amounts (46,15-17). Injection of particles is preferred to surgical implantation of larger devices such as films, discs, or rods. Large devices can be easier to remove from a patient if complications arise, but particles may also be recovered in surgery by scraping the subcutaneous area after administration of a local anesthetic. Particles were also shown to be less inflammatory than films [18].

Polymeric prodrug systems are composed of an active agent covalently bound to a polymeric backbone through a labile bond. These systems can be soluble for site-specific delivery or insoluble for systemic delivery of active agents. Five major design considerations are important in tailoring a polymeric prodrug system to give the desired release characteristics: (i) hydrophilic character and molecular weight of the polymer, (ii) type and length of spacer between drug and polymer, (iii) lability of the covalent linkage, (iv) initial drug loading, and (v) particle size or geometry of the device. The drug release rate from the designed system is controlled by one or more of the following processes: water permeation, hydrolysis of the labile drug-polymer bond, drug diffusion or degradation of the matrix. In general, the rate of hydrolysis will be slower than the rates of water permeation and drug diffusion and the release rate is expected to be governed by the rate of hydrolysis. Insoluble polymeric prodrugs of contraceptive steroids have exhibited long-term constant release both in vitro and in vivo $[15,17]$.
Preparation of biodegradable copolymer of $N^{5}(3-$ hydroxypropyl)-L-glutamine and leucine as drug carrier

Biodegradable devices possess all of the advantages of nondegradable systems such as longterm delivery capability, controlled delivery and increased patient compliance [19]. A unique advantage of biodegradable devices is elimination of the need for surgical removal of the device after depletion of the drug.

Poly (amino acids) represent a class of biodegradable polymers with a wide variety of physical and chemical properties which can be manipulated to tailor a specific system. Due to the wide range of amino acids commercially available, many different polymers and copolymers can be synthesized. Synthetic poly ( $\alpha$-amino acids) are known to be biodegradable [20]. Those poly ( $\alpha$ amino acids) that have shown the greatest potential utility in designing drug delivery systems are homopolymers of L-glutamic acid or L-aspartic acid and copolymers of these amino acids with L-leucine and L-valine [21]. The polymers of these amino acids contain free carboxyl groups on the pendant side chains. These side chains may be modified by esterification or amidation to make a nonionizable and more hydrophobic derivative [7,8]. Particularly useful has been the development of poly(hydroxyalkyl-L-glutamines) in which the side chain has been extended and rendered nonionizable while retaining a hydrophilic group [8]. The pendant side chains have been modified by covalent coupling of bioactive agents through hydrolytically labile linkages to give polymeric prodrugs [4-6, 15-17].

In our previous studies copolymers of HPG and Leu with varying amounts of Leu were used as the carrier in disc- and particle-shaped naltrexone conjugates $[4,5]$. By increasing the content of Leu residues from 10 to $50 \%$ the in vitro drug release rate from particles was observed to decrease, which is probably due to the decreased water permeability of the polymer-drug conjugate [5]. However, even with $50 \%$ incorporation of Leu residues the device was exhausted after 15 days [5]. To enhance the duration of 
drug release the 50/50 HPG/Leu copolymer was chosen for the preparation of conjugates with a hydrolytically more stable drug-polymer bond [6]. This copolymer has been shown to undergo degradation in vitro [20].

A random copolymer of $\gamma$-benzyl-L-glutamate (BLG) and Leu was prepared by polymerisation of an equimolar mixture of the amino acid $\mathrm{N}$ carboxyanhydride monomers in dioxane using TEA as the catalyst according to the method of Blout et al. [10]. Treatment of the copolymer with 3-aminopropanol for 5 days yields the HPG/Leu copolymer. The molecular weight of each polymer was determined by viscometry and WALS (Table 1). From WALS absolute molecular weight values (Mw) of 134000 (BLG/Leu copolymer) and 76100 (HPG/Leu copolymer) were obtained. The viscosity values agree reasonably well with the WALS results although the incorporation of Leu into the polymers does not allow exact viscometric comparisons with the literature data obtained for the homopolymers PBLG and PHPG [8,22].

\section{Preparation and in vitro hydrolysis of naltrexone monoacetate prodrugs}

Naltrexone has two groups for possible bond formation, the 3-phenolic and 14-tertiary hydroxyls (see Fig. 1). In order to facilitate selective coupling of the drug with the polymeric carrier the 3-acetate (N3A) and 14-acetate (N14A) derivatives of naltrexone were synthesized. N3A was prepared as described previously [4]. N14A was prepared by selective hydrolysis of naltrexone-3,14-diacetate (N3,14A) [11] with diluted sulfuric acid as described for naloxone-3,14-di-

TABLE 1

Copoly ( $\alpha$-amino acid $)$ molecular weights

\begin{tabular}{|c|c|c|c|c|c|}
\hline Copolymer & $\begin{array}{l}\left(\eta_{\mathrm{sp}} / \mathrm{c}\right)_{0.2 \%} \\
(\mathrm{dl} / \mathrm{g})\end{array}$ & {$[\eta]$} & $\begin{array}{l}\mathrm{dn} / \mathrm{dc} \\
\left(\mathrm{cm}^{3} / \mathrm{g}\right)\end{array}$ & $\begin{array}{l}\text { Mv } \\
\left(\times 10^{-3}\right)\end{array}$ & $\begin{array}{l}\text { Mw } \\
\left(\times 10^{-3}\right)\end{array}$ \\
\hline P(BLG ${ }^{50} /$ Leu $\left.^{50}\right)$ & 0.96 & 0.97 & 0.058 & $145^{\mathrm{m}}$ & 134 \\
\hline $\mathrm{P}\left(\mathrm{HPG}^{50} / \mathrm{Leu}^{50}\right)$ & 0.62 & 0.72 & 0.084 & $60.3^{b}$ & 76.1 \\
\hline
\end{tabular}

- Estimated from [ $\eta$ ] vs. molecular weight plot of Mitchell et al. [22]

bstimated from [ $\eta$ ] vs. molecular weight plot of Lupu-Lotan et al. [8]. acetate [23]. The compounds were found to be homogenous as judged from the sharp melting point and from TLC and HPLC. The chemical structure of each monoacetate was confirmed by ${ }^{1} \mathrm{H}$ and ${ }^{13} \mathrm{C}$ NMR spectroscopy $[4,6]$.

The conversion of both monoacetate prodrugs back to naltrexone was accomplished by hydrolysis in phosphate-buffered saline at $\mathrm{pH} \mathrm{7.4.} \mathrm{To}$ show that generation of parent drug will occur if an ester derivative is released from the polymerdrug conjugate the rates of ester hydrolysis were assessed. The first-order rate constant for the hydrolysis of the phenolic acetate ester to parent drug $\left(k_{3-\mathrm{OAc}}=6.84 \times 10^{-4} \mathrm{~min}^{-1}\right)$ is approximately an order of magnitude less than that seen for the tertiary ester $\left(k_{14-\mathrm{OAc}}=7.62 \times 10^{-3}\right.$ $\min ^{-1}$ ) under the conditions employed. The surprisingly fast hydrolysis of the 14-ester may be explained by intramolecular catalysis by the nitrogen lone pair and the electron withdrawing nature of the nitrogen (especially when protonated, $\mathrm{p} K_{\mathrm{a}}=8.13$ ) resulting in an electron-deficient carbonyl carbon.

The relatively slow conversion of the 3-acetate may not present a problem with regards to bioactivity, as it has been shown that naloxone-3-acetate has $60 \%$ greater activity in dogs than the parent drug [23]. Naloxone is a narcotic antagonist structurally similar to naltrexone having an $N$-allyl group rather than an $N$-cyclopropylmethyl group at the 17 position. Similarly, the release of naltrexone 14-acetate is acceptable in view of the enhanced bioactivity of naloxone-14acetate compared with naloxone. Interestingly, the diacetate of naloxone actually demonstrated decreased activity versus the parent drug [23]. The half-life of the 14-acetate ester in PBS is about $90 \mathrm{~min}$, while that of the phenolic acetate is $1000 \mathrm{~min}$. It is likely that ester hydrolysis rates in vivo would be faster due to the presence of non-specific esterases in plasma. Salicylate esters of the naltrexone phenolic hydroxyl moiety were shown to have plasma half lives of about 30 min in dogs and humans [24].

\section{Preparation of polymeric naltrexone conjugates}

In previous studies naltrexone release from polymeric conjugates required the hydrolysis of 
ester bonds between drug and carrier [4,5]. In view of the relatively fast hydrolyses of the monoacetate prodrugs of naltrexone we anticipated that linkage of the drug with the polymer across a hydrolytically more stable carbonate bond may result in a slower rate and a prolonged duration of drug release.

The synthetic scheme for coupling of naltrexone monoacetates to hydroxyl-containing polymers using a carbonate bond is outlined in Fig. 1.

Naltrexone-3 acetate (N3A) and naltrexone14-acetate (N14A) were reacted with triphosgene in the presence of triethylamine (TEA). TEA was chosen as the base catalyst due to its demonstrated superiority over other bases (pyridine, lutidine, 4-dimethyl-aminopyridine and dimethylformamide) in model reactions. The resultant chloroformate of each monoacetate was reacted with $\mathrm{P}\left(\mathrm{HPG}^{50} / \mathrm{Leu}^{50}\right)$ in situ to give a carbonate-bound drug-polymer conjugate.

The carbonate bound conjugates were loaded with $11.3 \%(w / w) .(N 3 A)$ and $8.4 \%(w / w)$ (N14A), respectively. The low drug loading of the conjugates is tentatively explained by the extreme sensitivity of the chloroformate intermediates to traces of water in the reaction mixture.

\section{In vitro release characteristics of polymeric naltrexone conjugates}

The polymeric prodrugs can be fashioned into various device forms affecting the rate of drug release. Particulate devices will generally release drug in a non-constant declining rate in vitro. However, the boundary layers that can exist in vivo may provide for constant drug release. Earlier work utilized discs and particles of various
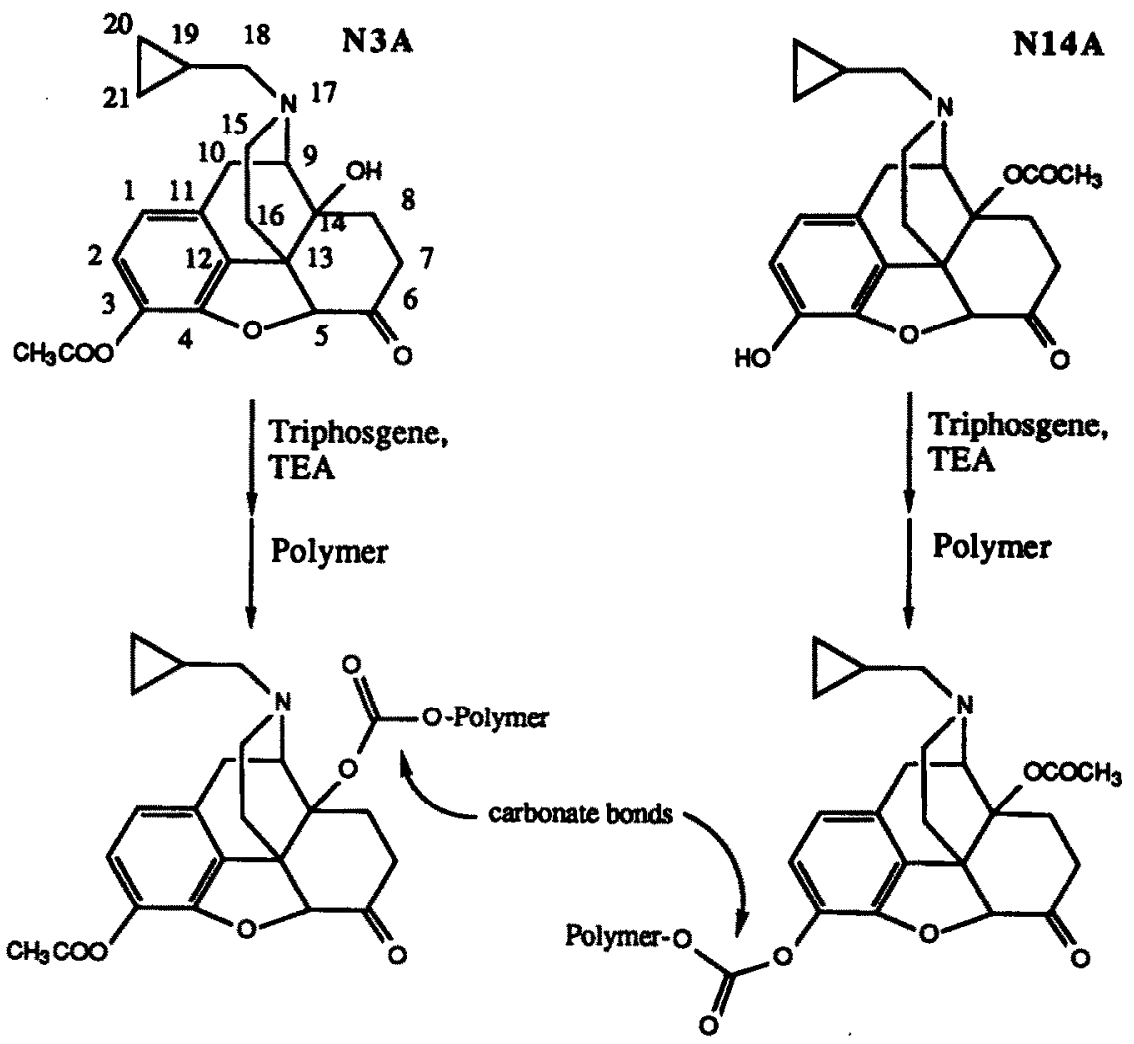

N14A

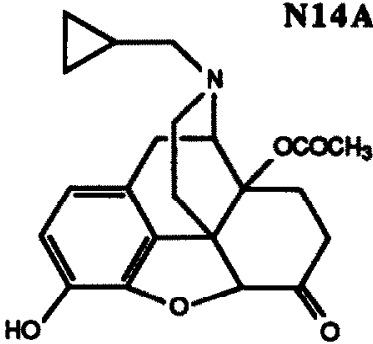

Triphosgene, Polymer 
HPG/Leu copolymers coupled to naltrexone via ester linkages [4-6]. The carbonate-bound conjugates of N3A or N14A and P(HPG $\left.{ }^{50} / \mathrm{Leu}^{50}\right)$ were ground and passed through sieves yielding particles of various size ranges to study the influence of total surface area on drug release.

Figure 2 shows the cumulative naltrexone release with time from the N14A-bound conjugate in which coupling was effected through the phenolic 3-OH. A relatively large burst is observed for all particle sizes up to a cumulative fraction of released drug equal to $0.2-0.4$ dependent on the size range. After the burst the release rate becomes almost constant for each size range. Smaller particles release drug more quickly than larger particles both during the burst phase and afterwards. Release rates after the initial burst averaged $49.7,33.9,30.1$, and $24.5 \mu \mathrm{g} /$ day $/ 100$ $\mathrm{mg}$ conjugate for the particles sized 20-50, 50$100,100-200$, and $200-350 \mu \mathrm{m}$, respectively.

Particles of the conjugate of N3A having a carbonate bond between the tertiary 14-OH and the polymer also displayed a slight burst followed by near constant release of naltrexone. The cumulative fraction drug release from these various sized systems is plotted versus time in Fig. 3.

Particle size effects similar to the N14A carbonate bound conjugate were observed. The naltrexone release rates following the burst period averaged $110.9,102.5,87.4$, and $79.0 \mu \mathrm{g} /$ day/ $100 \mathrm{mg}$ conjugate for the particle size series 20 $50,50-100,100-200$, and 200-350 $\mu \mathrm{m}$. These

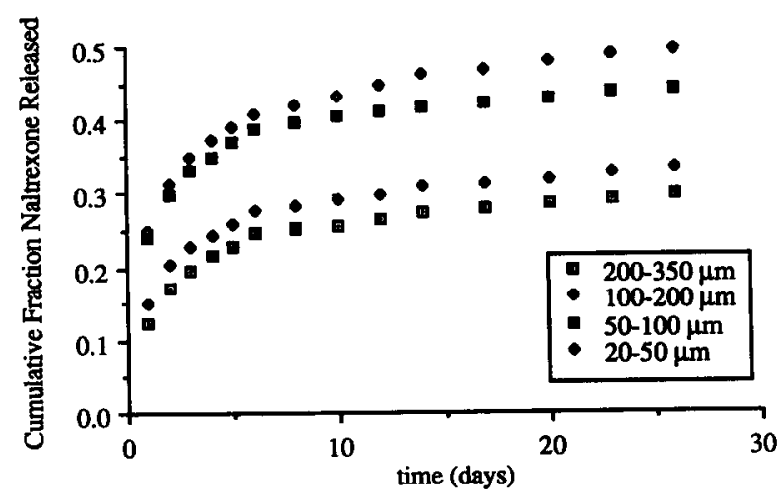

Fig. 2. Cumulative fraction naltrexone released from N14Acopoly $\left(\mathrm{HPG}^{50} / \mathrm{Leu}^{50}\right.$ ) conjugate particles of various sizes.

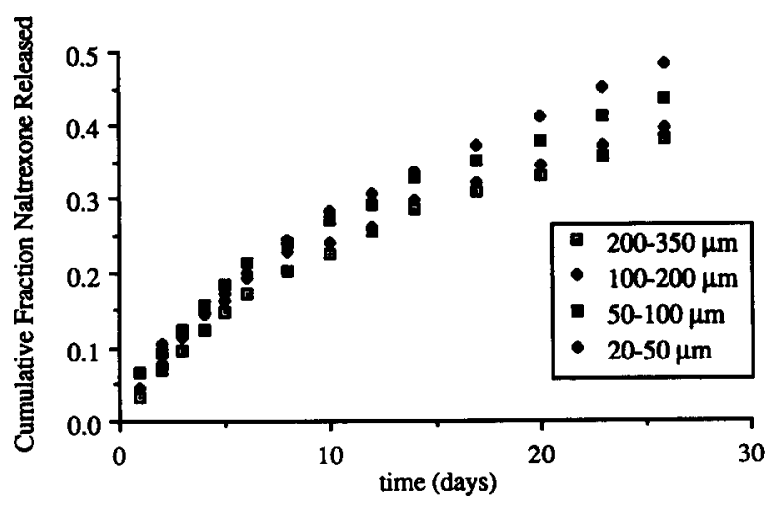

Fig. 3. Cumulative fraction naltrexone released from N3Acopoly $\left(\mathrm{HPG}^{50} / \mathrm{Leu}^{50}\right)$ conjugate particles of various sizes.

rates are at least two times greater than those seen for the N14A conjugate, which may be explained by an enhanced hydrolytic lability of the carbonate bond at position 14 of the drug similar to that observed for the monoacetates.

The carbonate-bound naltrexone conjugates were obtained with much lower drug loadings than previously studied ester-bound conjugates preventing a direct comparison of the effect of linkage type. The much lower rates of naltrexone release observed for the carbonate-bound conjugates may be due to lower drug loading, bond type, or a combination of both factors.

The burst effect observed for both conjugates in the initial stages of release may be due to physically adsorbed drug, an excess of drug moieties oriented outwards at the surface, edge effects, hydrophilic impurities acting as swelling enhancers, or copolymer chains with higher HPG content which solvate faster and release the drug more quickly.

\section{In vivo release characteristics of polymeric naltrexone conjugates}

The carbonate-bound polymeric prodrugs of naltrexone were tested in vivo by examination of their plasma naltrexone profiles after subcutaneous injection of the conjugate particles in rats. 
Plasma was analyzed by GC/MS and the concentrations were normalized to volume of sample obtained and amount of conjugate injected. The analytical method only provided for quantitation of free naltrexone excluding analysis of the acetate prodrugs.

The conjugates of naltrexone monoacetates demonstrated burst effects in vivo (Figs. 4 and 5).

Plasma levels in the early stage of delivery were between 2 and $4 \mathrm{ng} / \mathrm{ml} / \mathrm{mg}$ conjugate for both the N3A and N14A devices. After the burst plasma naltrexone levels averaged $1.03 \pm 0.52$

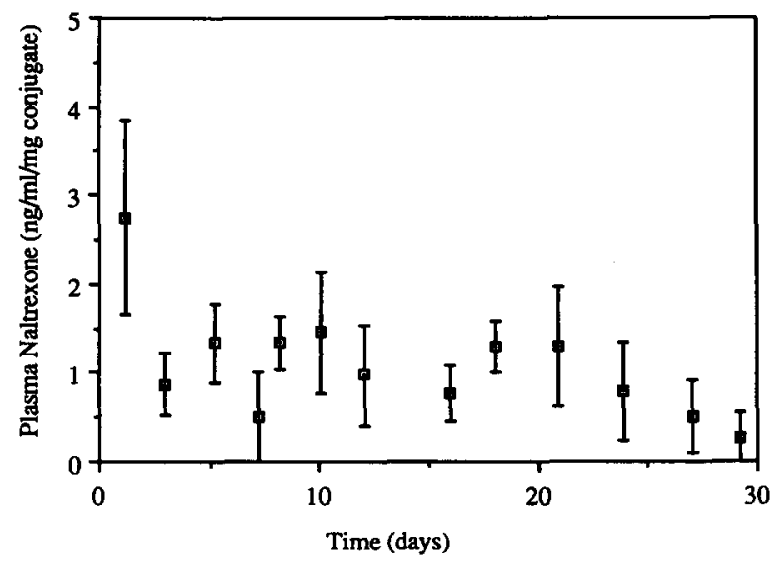

Fig. 4. Plasma naltrexone levels following injection of N14Acopoly $\left(\mathrm{HPG}^{50} / \mathrm{Leu}^{50}\right)$ conjugate particles $(50-100 \mu \mathrm{m})$ subcutaneously in rats $(n=3)$.

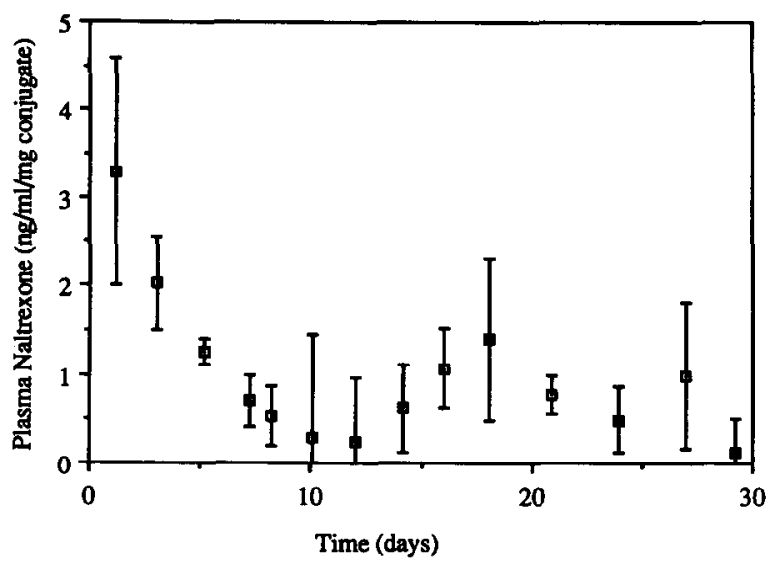

Fig. 5. Plasma naltrexone levels following injection of N3Acopoly $\left(\mathrm{HPG}^{50} / \mathrm{Leu}^{50}\right)$ conjugate particles $(50-100 \mu \mathrm{m})$ subcutaneously in rats $(n=3)$. $\mathrm{ng} / \mathrm{ml} / \mathrm{mg}$ conjugate injected for the N14A polymeric prodrug and $0.70 \pm 0.39 \mathrm{ng} / \mathrm{ml} / \mathrm{mg}$ conjugate injected for the N3A conjugate.

Bursts may be caused by physically adsorbed drug and surface effects, but are also likely to be due to the conditions present within the subcutaneous site. An initial period of inflammation is common, and this is accompanied by a decrease in local pH. Rapid infiltration of the implant site by macrophages can provide for an increased level of enzyme activity which can also contribute to any burst. Similarly, the periods of more constant release may be influenced by the formation of a fibrous capsule around the particles.

Assuming that the volume of distribution of naltrexone per kilogram of body weight is of the same order of magnitude for both rats and humans, similar plasma levels in humans could possibly be realized with subcutaneous injections of $100-500 \mathrm{mg}$.

\section{Conclusions}

In the present study the preparation as well as in vitro and in vivo drug release characteristics of naltrexone conjugates of a biodegradable copolymer of $N^{5}$-(3-hydroxypropyl)-L-glutamine and L-leucine was investigated. By linking naltrexone monoacetates at either the 3- or 14-position through a carbonate bond with the polymeric carrier the in vitro drug release rate from particles was favourably suppressed compared with ester-bound conjugates studied previously $[4,5]$. The in vivo release of drug in rats showed therapeutically promising values of the mean plasma level for at least one month. The conjugate design may be improved by achieving higher drug loads and by diminishing the in vitro and in vivo burst effects. The results warrant a more detailed study of the in vitro and in vivo mechanism of drug release from the polymeric conjugates.

\section{Acknowledgement}

This work has been supported by NIH Grants DA-02391 and GM-36669 (new HL-44539). 


\section{References}

1 C.N. Chiang, L.E. Hollister, A. Kishimoto and G. Barnett, Kinetics of naltrexone sustained-release preparation, Clin. Pharmacol. Ther., 36 (1984) 704-708.

2 C.P. O'Brien, R. Greenstein, J. Ternes and G.E. Woody, Clinical pharmacology of narcotic antagonists. In: B. Kissin, J.H. Lowinson and R.B. Millman (Eds.), Recent Developments in Chemotherapy of Narcotic Addiction, Ann. N.Y. Acad. Sci., 311 (1978) 232-240.

3 S. Archer, Design consideration for long-acting antagonists. In: M.C. Braude, L.S. Harris, E.L. May, J.P. Smith and J.E. Villareal (Eds.), Narcotic Antagonists (Advances in Biochemical Psychopharmacology, Vol. 8), Raven Press, Nw York, 1973, pp. 549-553.

4 N. Negishi, D.B. Bennett, C.S. Cho, S.Y. Jeong, W.A.R. Van Heeswijk, J. Feijen and S.W. Kim, Coupling of naltrexone to biodegradable poly ( $\alpha$-amino acids), Pharm. Res., 4 (1987) 305-310.

5 D.B. Bennett, N.W. Adams, X. Li, J. Feijen and S.W. Kim, Drug-coupled poly (amino acids) as polymeric prodrugs, J. Bioact. Compat. Polym., 3 (1988) 44-52.

6 D.B. Bennett, Biodegradable Polymeric Prodrugs of Naltrexone, Ph.D. dissertation, University of Utah, 1990.

7 W.L. Sederel, A. Bantjes and J. Feijen, A route to anionic hydrophilic films of copolymers of L-leucine, L-aspartic acid and L-aspartic acid esters, Polymer, 16 (1975) 735-738.

8 N. Lupu-Lotan, A. Yaron, A. Berger and M. Sela, Conformational changes in the nonionizable watersoluble synthetic polypeptide poly- $N^{5}$-(3-hydroxypropyl)-Lglutamine, Biopolymers, 3 (1965) 625-655.

9 G.D. Fasman, C. Lindblow and E. Bodenheimer, Conformational studies on synthetic poly- $\alpha$-amino acids. Factors influencing the stability of the helical conformation of poly-L-glutamic acid and copolymers of L-glutamic acid and L-leucine, Biochemistry, 3 (1964) 155166.

10 E.R. Blout, R.H. Karlson, P. Doty and B. Hargitay, Polypeptides, I. The synthesis and the molecular weight of high molecular weight polyglutamic acids and esters, J. Am. Chem. Soc., 76 (1954) 4492-4493.

11 W.L. Dewey, L.S. Harris, J.F. Howes and J.A. Nuite, Effect of various neurohumoral modulators on the activity of morphine and the narcotic antagonists in the tailflick and phenylquinone tests, J. Pharmacol. Exp. Ther., 175 (1970) 435-442.

12 G.A. Koolpe, W.L. Nelson, T.L. Gioannini, L. Angel and E.J. Simon, Diastereomeric 6-deoxy-6-spiro- $\alpha$-methylene- $\gamma$-butyrolactone derivatives of naltrexone and oxymorphone. Selective irreversible inhibition of naltrex- one binding in an opioid receptor preparation by a conformationally restricted Michael acceptor ligand, J. Med. Chem., 27 (1984) 1718-1723.

13 C. Thies, Characterization of microcapsules containing naltrexone or naltrexone pamoate. In: D.A.R. Paul and F.W. Harris (Eds.), Controlled Release Polymeric Formulations, American Chemical Society, Washington, DC, 1976, pp. 190-194.

14 K.M. Monti, R.L. Foltz and D.M. Chin, Analysis of naltrexone and $6-\beta$-naltrexone in plasma and urine by gas chromatography/negative ion chemical ionization mass spectrometry, J. Anal. Toxicol,, in press.

15 J.M. Anderson, In vitro and in vivo studies of drug-releasing poly (amino acids). In: D.A. Tirrel, L.G. Donaruma and A.B. Turek (Eds.), Macromolecules as Drugs and as Carriers for Biologically Active Materials, Ann. N.Y. Acad. Sci., 446 (1985) 67-75.

16 K.R. Sidman, A.D. Schwope, W.D. Steber, S.E. Rudolph and S.B. Poulin, Biodegradable, implantable sustained release systems based on glutamic acid copolymers, J. Membr. Sci., 7 (1980) 277.

17 R.V. Petersen, C.G. Anderson, S.M. Fang, D.E. Gregonis, S.W. Kim, J. Feijen, J.M. Anderson and S. Mitra, Controlled release of progestins from poly ( $\alpha$-amino acid) carriers. In: R. Baker (Ed.), Controlled Release of Bioactive Materials, Academic Press, New York, 1980, pp. 45-61.

18 S. Yolles, T.D. Leafe and F.J. Meyer, Timed-release depot for anticancer agents, J. Pharm. Sci., 64 (1975) 115.

19 D.A. Wood, Biodegradable drug delivery systems, Int. J. Pharm., 7 (1980) 1-18.

20 T. Hayashi, Y. Tabata and A. Nakajima, Biodegradation of poly ( $\alpha$-amino acid ) in vitro, Polym. J., 17(3) (1985) 463-471.

21 R.V. Petersen, Biodegradable drug delivery systems based on polypeptides. In: C.G. Gebelein and C.E. Carraher (Eds.), Bioactive Polymeric Systems: An Overview, Plenum Press, New York, 1985, pp. 151-177.

22 J.C. Mitchell, A.E. Woodward and P. Doty, Polypeptides, XIV. The polydispersity and configuration of low molecular weight poly- $\gamma$-benzyl-L-glutamates, J. Am. Chem. Soc., 79 (1957) 3955-3960.

23 C. Linder and J. Fishman, Narcotic antagonists, 1. Isomeric sulfate and acetate esters of naloxone ( $N$-allylnoroxymorphone), J. Med. Chem., 16 (1973) 553-556.

24 M.A. Hussain and E. Shefter, Naltrexone-3-salicylate (a prodrug of naltrexone): synthesis and pharmacokinetics in dogs, Pharm. Res., 5 (1988) 113-115. 